Book Review

\title{
The Semiotics of Emoji: The Rise of Visual Language in the Age of the Internet
}

\author{
Laura Kerslake and Rupert Wegerif* \\ Faculty of Education, University of Cambridge, Cambridge, CB2 8PQ, UK; E-Mails: laurakerslake@gmail.com (L.K.), \\ rw583@cam.ac.uk (R.W.) \\ * Corresponding author
}

Submitted: 14 May 2017 | Accepted: 7 June 2017 | Published: 21 December 2017

\begin{abstract}
This paper reviews Marcel Danesi's new book on the use of emoji in particular, and the use of visual language more generally. Danesi offers a number of interesting examples of emoji use, pointing out that their use has risen considerably in a number of contexts. He goes on to question how far emoji use can be extended by examining the structure of the emoji 'language'. Overall this is an accessible book that presents a number of examples of visual languages and comments on the possibilities and challenges for visual language use against a backdrop of technological change.
\end{abstract}

\section{Keywords}

emoji; visual communication; semiotics; visual languages

\section{Issue}

This book review is part of the issue "Visual Communication in the Age of Social Media: Conceptual, Theoretical and Methodological Challenges", edited by Uta Russmann (FHWien der WKW University of Applied Sciences for Management \& Communication, Austria) and Jakob Svensson (Malmö University, Sweden).

(C) 2017 by the authors; licensee Cogitatio (Lisbon, Portugal). This article is licensed under a Creative Commons Attribution 4.0 International License (CC BY).

\section{Background}

As this book points out in its opening sentence, the 'face with tears of joy' emoji became the Oxford English Dictionary (OED) Word of the Year in 2015. The Word of the Year is chosen as the best reflection of that year in a single word-other contenders included refugee and Brexit (OED, 2016). The OED justification for its choice reveals a great deal about the use of emoji in current digital communication; the OED website states that it made the decision to give the Word of the Year distinction to an emoji because use of emoji not only increased considerably in 2015-with the 'face with tears of joy' emoji accounting for a fifth of all emoji used-but was also used broadly across social groups. This trend saw emoji used increasingly by advertisers and politicians.

The term emoji refers specifically to the characters systematised by Unicode, which has allowed emoji to be standardised across operating systems (p. 4). The term emoji actually means $e$ (picture) moji (letter/character) (p. 2), but its similarity to the English word emotion is of particular note because that is, in fact, how most people use emoji. The designer of emoji, Shigetaka Kurita, highlights this point, stating that it is the rise of digital communication, a medium which doesn't readily allow for expressions of emotion, which made the use of emoji necessary to compensate for the inability to convey facial expression, tone of voice and gesture in digital communication (Lucas, 2016).

\section{This Book's Place}

The OED website also adds that emoji can 'cross language barriers' (OED, 2016), which is a central theme of this book. Whether or not emoji can be considered a universal language is an issue which is raised in each of the chapters in the book as Marcel Danesi considers both so- 
cial and communicative elements of emoji use, as well as linguistic considerations.

There is a theoretical aspect to each chapter which is mostly light in order to maintain accessibility to the general audience to which this book aims to appeal. Despite this there are a number of technical linguistic terms used, for example 'phatic' which refers to a communication which contains emotive content (p. 18). These are used sparingly, however, and with full explanation, and so in its mix of general and specialised language, the book does manage to command more general appeal whilst situating its explanations within an established linguistic context.

While the focus of the book is emoji, the sub-title The Rise of Visual Language in the Age of the Internet indicates the breadth of its scholarship. It is an extended essay on visual languages with many interesting diversions exploring a wide range of relevant topics such as the possible role of gesture in the evolution of language, Medieval illuminated manuscripts, rebus writing, Leibniz's attempt to develop a universal visual language and even Teilhard de Chardin's account of an emerging global consciousness written, of course, well before the Internet Age. This historical perspective enables Danesi to advance the interesting hypothesis that emoji may be a retrieval of hybridity in writing, that is to say the combination of picture writing with phonetic writing, where this hybridity goes back to the origins of writing and has been a recurrent theme in the history of writing. He also suggests, in an interesting excursion on Derrida, that emoji writing might be seen as an 'anti-hegemonic' reaction to the power relations of print literacy.

Research methods are not explicitly set out in the book, although its origin was based on a database of emoji communications compiled by four research students at the University of Toronto. Instead, examples of the communications are used throughout the book, together with questions and participant responses. There is occasion when it would have been useful to know more about the research context. For example, one of the questions related is 'Does emoji writing make you feel more comfortable when communicating, reducing the stress of face-to-face interaction?' (p. 177). We are then told that everyone answered in the affirmative, yet that is hardly surprising given that it appears to be a leading question, making an assumption that face-to-face communication is stressful.

The book is, for the most part, well produced and easy to use with a full index. If there is a practical criticism of this book, it is that the examples of texts and digital communications containing emoji are in small print on a coloured background, making them very difficult to see. It is therefore necessary to refer to accompanying text and back to the emoji frequently in order to work out what is being referred to, which is somewhat frustrating. There are, however, numerous examples provided to illustrate the author's points, offering an interesting array of the uses of emoji.

\section{Emoji as Universal Language}

The question of whether emoji are a complement to the written word or a substitute is an important one for what has been described as the world's fastest growing language (Cioletti, 2016). Chapter Three-'Emoji Competence' - considers this issue, telling us that there are two types of emoji text, adjunctive, where emoji are used alongside text, and substitutive, where emoji are used wholly in place of text.

The two examples given by the author in fact serve as illustrations as to why emoji use-with or without accompanying words-does not (yet) seem sufficient to produce texts that are equivalent in terms of meaning to the written word alone. The first (p. 36) is an entire, substitutive, emoji text entitled 'My birth story'. The author points out that it is generally intelligible, but then goes on to misinterpret it, stating that it tells of the writer's feelings 'when she was born' (p. 38). This isn't the case-the phrase 'My birth story' is understood to refer to a woman's story of when she gave birth. The website BabyCentre has a thread asking its group members to post their birth stories in emoji. A look at this highlights the issue of experience in emoji competence. Once one has read a number of these emoji birth stories, then they become easier to decode (time waiting, drugs taken etc). If one has had the experience of giving birth, then the emoji which represent these events are much easier to decode. It doesn't seem likely that, with any degree of emoji competence, this would be decodable without knowledge of the experience. This is a challenge to the notion of emoji as a language (let alone a universal one), as the traditional written word is capable of clearly expressing experiences which one has not had.

The same issue is also highlighted in the second example given by Danesi in this chapter. This example is an adjunctive one, the first sentence of this reads 'This is a [book emoji] all about how my life got [arrow of circles emoji] [up arrow emoji] [down arrow emoji]' ( $p$. 38). Danesi again highlights that this can be easily understood and translated as 'this is a story about how my life got turned around, up and down' (p. 39). But, as is clear from the rest of the example given, it is in fact a partial emoji representation of the theme song to the television show The Fresh Prince of Bel Air. The first line of the song is 'Now this is a story all about how/my life got flipped turned upside down' (Smith and Townes, as cited on the LyricsFreak website). It has a very specific meaning that is not translatable as anything else.

There is, then, an extent to which emoji competence is synonymous with cultural competence, and as such, this does not provide a compelling indication that any degree of emoji competence is sufficient to decode emoji text. Instead it highlights the point that, as there are no native emoji speakers, instead the ways in which emoji are used are currently grounded in first languages. 'No special training is needed to learn emoji use' (p. 158). The examples given here, and the existence of the Emoji- 
pedia, which provides a reference guide to the meanings of emoji indicate that training is needed to learn emoji use. Despite this, Danesi is correct that people use emoji in large numbers on a day-to-day basis without the need for any formal instruction.

\section{The Possibilities of Universal Languages}

One of the interesting aspects of this book is the array of other picture-writing systems referenced, for example Pitman Shorthand, of which many people will have heard, and Blissymbolics, which remains more obscure. The latter was invented in the mid twentieth century by Charles Bliss, and is interesting because it is capable of conveying whole sentences.

The example of Blissymbolics perhaps provides a hint as to why a universal writing system is an ideal which can never be realised. While it provides a more comprehensive communication system than emoji, Bliss himself was determined that his symbols had a specific meaning, and was vitriolic when institutions interpreted them in other ways. More recently, The Fundamental Rules of Blissymbolics document states that 'Creation of terminology must be carried out according to the fundamental rules of Blissymbolics to ensure the integrity of the system' (Blissymbolics Communication International, 2004, p. 2).

Emoji, while standardised by Unicode, does not follow this pattern. Anyone can propose new emoji to become part of the canon and even where icons are rejected, existing emoji take on new meanings to fill this gap. An example of this is the aubergine emoji, which is commonly understood as a phallic symbol. A quick online search reveals a plethora of articles from popular media indicating to readers popular versus official meanings of emoji. (e.g. Good Housekeeping Guide, 2016). This raises interesting questions about Danesi's claim that 'human communication in written form is evolving more and more on a single path of hybridization' ( $p$. 88). Emoji as language is both a global practice which highlights the role of human technology in transcending national boundaries, and at the same time a culturally situated practice in flux; emoji use therefore calls attention to universal and particular instances of lan- guage. The hybridization of visual languages with written ones augments human possibilities for universal language development.

\section{Conclusion}

Ultimately, as is reiterated throughout this book, emoji are primarily used to add emotion to digital communications. They tend to add a positive tone to messages, seen by their users as 'fun' (p. 179). This is an important compensation for the relative lack of contextual clues in online communication of the kind that are so important to guiding interpretation in face to face communication. Emoji used online can play a similar role to facial expression and gesture and are often to undermine seriousness with a sense of shared humanity. Danesi ends by stating that his conclusion is that he has no conclusion. He rightly points out that the adaptability of human communication is likely to prevail, and the continued use of emoji will depend considerably on the form that new technological development takes as much as any inherent value in emoji communication itself.

\section{Conflict of Interests}

The authors declare no conflict of interests.

\section{References}

Blissymbolics Communication International. (2004). The fundamental rules of Blissymbolics. Retrieved from http://www.blissymbolics.org/images/blissrules.pdf

Danesi, M. (2016). The semiotics of emoji: The rise of visual language in the age of the Internet. London: Bloomsbury Publishing.

Cioletti, A. (2016). A universal language. License! Global, 19(3), 194-197.

Lucas, G. (2016). The story of emoji. Creative Review, 36(5), 74-81.

Oxford English Dictionary. (2016, November 16). Oxford dictionaries word of the year 2015 is.... Retrieved from http://blog.oxforddictionaries.com/ 2015/11/word-of-the-year-2015-emoji

\section{About the Authors}

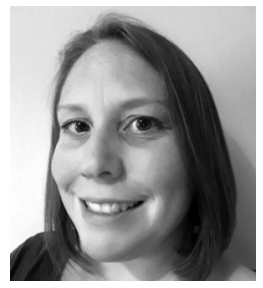

Laura Kerslake is conducting research into Philosophy with Children at the University of Cambridge. Her work looks at how philosophy with young children is augmented by the introduction of a pictorialbased talking framework to help to create a dialogic space in the classroom. Laura is editing a forthcoming book on the theory of teaching thinking, as well as having articles published in peer-reviewed journals. She is the creator of the Philosophize project and also lectures on philosophy courses at the University of Exeter. She is completing her PhD under the supervision of Rupert Wegerif. 


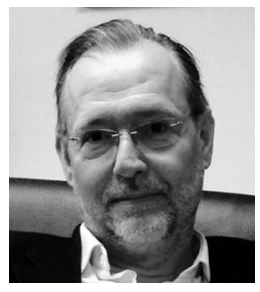

Rupert Wegerif is Professor of Education (2000) at the University of Cambridge. He did his PhD on Educational Technology at the Open University and his research focuses on dialogic education with technology in the context of the Internet Age. This includes a particular interest in the cognitive impact of new communications media (see 2013, Dialogic: Education for the Internet Age). He has gained over $£ 2$ million in research funding as principal investigator and published 9 books, as well as many highly cited peer-reviewed papers. He is founding lead editor of the Elsevier SSCl journal Thinking Skills and Creativity and is founder and co-convenor of the Educational Theory SIG of EARLI. 